\title{
Innovationen (forschend) gestalten - Zur neuen Rolle der Sozialwissenschaften
}

\author{
Jürgen Howaldt \\ Ralf Kopp \\ Michael Schwarz
}

Die innovationspolitische Debatte leidet unter folgenschweren Verkürzungen. Zum einen ist sie einseitig auf technologische Innovationen fokussiert, zum anderen wird Innovation als relativ einfacher, überschaubarer und politisch steuerbarer Prozess gesehen. Wie die sozialwissenschaftliche Innovationsforschung gezeigt hat, wird dies der Komplexität von Innovationsprozessen nicht gerecht - und übersieht obendrein die Bedeutungszunahme sozialer Innovationen. Beides aber prädestiniert die Sozialwissenschaften, die im praktischen Innovationsgeschehen bislang kaum eine Rolle spielen, hier eine zentrale, impulsgebende und koordinierende Funktion zu übernehmen. Es erfordert umgekehrt auch eine Neubestimmung ihrer aktiven Funktion in Innovationsprozessen. ${ }^{1}$

\section{Einleitung}

Vor nunmehr knapp 20 Jahren hat Wolfgang Zapf in der Zeitschrift Soziale Welt (Zapf 1989) die Bedeutung von sozialen Innovationen hervorgehoben und dabei drei wichtige, aber rückblickend betrachtet kaum weiter diskutierte Fragestellungen aufgeworfen und behandelt: Was sind soziale Innovationen, was kann Innovationstheorie über sozialen Wandel, Krisen und Steuerungsfähigkeit in modernen Gesellschaften erklären und was ist die Rolle der Sozialwissenschaften bei der Förderung sozialer Innovationen?

Diesen Anstoß von Zapf aufgreifend möchten wir versuchen, die sich unter den Bedingungen der Wissens- und Dienstleistungsgesellschaft abzeichnende Bedeutungszunahme sozialer Innovationen für die Konturierung eines soziologisch aufgeklärten, postindustriellen Innovationsparadigmas und für eine Neubestimmung der Rolle der Sozialwissenschaften im Innovationsprozess fruchtbar zu machen.

Unsere zentrale These lautet: Mit dem Übergang von der Industrie- zur Wissensund Dienstleistungsgesellschaft vollzieht sich ein „Paradigmenwechsel des Innovationssystems“ (Bullinger 2006, S. 14), in dessen Folge sich das Verhältnis von technologischen und sozialen Innovationen verändert. Zielte Innovation bisher primär darauf ab, mit den Mitteln der Natur- und Ingenieurwissenschaften neue Produkte und Verfahren hervorzubringen, werden künftig soziale Innovationen stärker in den Fokus rücken. Dies weckt einen Kompetenzbedarf, der prioritär von den Sozialwissenschaften gedeckt werden kann, sie aber zugleich mit neuen Herausforderungen konfrontiert, ihr Kompetenzprofil für soziale Innovationen zu schärfen.

Um diese These zu begründen, gehen wir zunächst auf den Beitrag der sozialwissenschaftlichen Innovationsforschung bei der Herausbildung eines soziologisch aufgeklärten, postindustriellen Innovationsverständnisses ein (Abschnitt 2). In einem zweiten Schritt wird die damit einhergehende zunehmende Bedeutung sozialer Innovationen begründet (Abschnitt 3). Am Beispiel von Projekten zum Thema „Wissensmanagement" arbeiten die Dysfunktionalitäten einseitig technologisch fokussierter Innovationsprozesse heraus und zeigen die sich abzeichnende Durchsetzung eines Innovationstyps sozial getriebener Technologieanpassung auf (Abschnitt 4). Abschließend fragen wir mit Zapfnach den Möglichkeiten und Herausforderungen dieser Entwicklungen für die Sozialwissenschaften, ihre Rolle im Innovationsprozess neu zu definieren (Abschnitt 5).

\section{Auf dem Weg zu einem soziologisch aufgeklärten Innovationsverständnis}

\subsection{THEMEN UND ERKENNTNISSE SOZIALWISSENSCHAFTLICHER INNOVATIONSFORSCHUNG}

Die sozialwissenschaftliche Innovationsforschung konzentriert sich aus unterschiedlichen Perspektiven und mit unter- schiedlichen Schwerpunktsetzungen auf die praktische Relevanz des Sozialen im und für den Prozess des Innovations-

\footnotetext{
Bei diesem Beitrag handelt es sich um die überarbeitete Fassung eines Vortrags auf der Herbsttagung der Sektionen Arbeits- und Industriesoziologie und Wissenschafts- und Technikforschung der Deutschen Gesellschaft für Soziologie zum Thema: „Innovationen und gesellschaftlicher Wandel ", Technische Universität Dortmund, 12./13. Oktober 2007
}

Jürgen Howaldt, Prof. Dr., ist Direktor der Sozialforschungsstelle Dortmund und Honorarprofessor an der Technischen Universität Dortmund. Arbeitsschwerpunkte: Organisationsentwicklung und-beratung, Netzwerkmanagement und Kooperationen, Wissenschafts-/Praxisverhältnis in der Soziologie. e-mail: howaldt@sfs-dortmund.de Ralf Kopp, Dr., ist Koordinator des Forschungsbereichs "Organisationsentwicklung und Beratung in der Netzwerkökonomie " der Sozialforschungsstelle Dortmund (ZWE der Technischen Universität Dortmund). Arbeitsschwerpunkte: Organisationswandel und -lernen, neue Formen der Wissensorganisation, Netzwerkmanagement, Innovationsforschung.

e-mail: kopp@sfs-dortmund.de Michael Schwarz, Dr., ist wissenschaftlicher Angestellter der Sozialforschungsstelle Dortmund (ZWE der Technischen Universität Dortmund) und Mitglied des Forschungsrates. Arbeitsschwerpunkte: Organisationswandel und -lernen, Organisations-, Netzwerk- und Politikberatung, Mikropolitik, Arbeits(zeit)politik, Nachhaltiges Wirtschaften. e-mail: schwarz@sfs-dortmund.de 
geschehens. Im Zentrum stehen die sozialen Voraussetzungen und Einflussfaktoren für (vor allem technische) Innovationen, das Wechselverhältnis von technischen und sozialen Innovationen, von Innovationen und gesellschaftlicher Entwicklung, der institutionelle Kontext und die Interaktion der am Innovationsprozess Beteiligten, die Organisation von Innovationen in und zwischen Unternehmen, das Problem der Plan- und Steuerbarkeit sowie der Folgenunsicherheit. Stand noch bis in die 1980er Jahre hinein die Vorstellung eines klar abgrenzbaren, linear ablaufenden Vorgangs im Vordergrund, wird vor allem durch Beiträge aus der sozialwissenschaftlichen Innovationsforschung und Techniksoziologie deutlich, dass man es bei Innovation mit einem komplexen sozialen Prozess zu tun hat. Die zunächst stark durch eine inhaltliche Fokussierung auf technischen Wandel und Technikgenese zwischen Pfad- ${ }^{2}$ und Kontextabhängigkeit ${ }^{3}$ geprägte sozialwissenschaftliche Innovationsforschung erhält durch die Theorie der reflexiven Modernisierung sowie das Leitbild der nachhaltigen Entwicklung bzw. die interdisziplinäre Nachhaltigkeitsforschung neue Impulse. Die Aufmerksamkeit richtet sich zunehmend auf neue intraund interorganisationale Verhandlungssysteme, Regulationsstrukturen, intermediäre Arrangements und GovernanceStrukturen - verstanden als notwendige soziale Innovationen (Heidenreich 1997). Fichter et al. (2006) definieren Nachhaltigkeitsinnovationen als „Durchsetzung solcher technischen, organisatorischen, nutzungssystembezogenen, institutionellen oder sozialen Neuerungen, die zum Erhalt kritischer Naturgüter und zu global und langfristig übertragbaren Wirtschaftsund Konsumstilen und -niveaus beitragen.“ Welche Innovation „als nachhaltig gelten darf und welche nicht, ist keine Frage der individuellen Bewertung, sondern eine Frage der kollektiven Attributierung von Nachhaltigkeit und damit das Ergebnis eines gesellschaftlichen Bewertungsprozesses" (ebd. S. 44f.).

Kennzeichnend für die neuere sozialwissenschaftliche Innovationsforschung ist vor allem, dass sie zunehmend die Vielzahl und Heterogenität der am Innovationsprozess beteiligten Akteure, Organisationen und Institutionen thematisiert. Damit ist eine Schwerpunktverlagerung auf Netzwerke und (nationale, regionale, lokale) Innovationssysteme, auf neue Formen der
Innovation, wie z. B. open innovation und open source (Chesbrough 2003; Reichwald/Piller 2005) verbunden, die auf die Kommunikation mit Wissensträgern aus Wirtschaft, Bildung, Politik und eine aktive Rolle der Nutzer bzw. Endverbraucher im Innovationsprozess setzen. Inhaltlich treten Themen wie Netzwerksteuerung, neue Formen der Wissensproduktion und -logistik, Prozesse des koevolutionären Lernens, transdisziplinäre Kommunikations- und Kooperationsbeziehungen als Forschungsfelder in den Vordergrund (Heidenreich 1997, S. 177; Nowotny et al. 2001).

\subsection{ANZEICHEN EINES \\ PARADIGMENWECHSELS IM INNOVATIONSSYSTEM}

Mit ihren Erkenntnissen hat die sozialwissenschaftliche Innovationsforschung bis in die Programmatik der nationalen und europäischen Innovationspolitik hinein dazu beigetragen, ein erweitertes und komplexes Innovationsverständnis zu etablieren. Den damit vollzogenen Wandel im Innovationsdiskurs bringt Birgit Blättel-Mink in ihrem Kompendium der Innovationsforschung auf den Punkt: „Von der Abweichung zur Norm, vom Akteur zum System“ - allerdings, wie sie ergänzt, immer in Reaktion auf das tatsächliche Innovationsgeschehen, selten - wie noch bei Schumpeter - „proaktiv“ (Blättel-Mink 2006, S. 12).

Als zentrale Elemente eines soziologisch aufgeklärten Innovationsverständnisses lassen sich zusammenfassend festhalten: Der systemische und soziale, nicht allein auf technische und organisatorische Neuerungen zu reduzierende Charakter von Innovationen sowie die Aspekte Komplexität, Risikohaltigkeit und Reflexivität, Nichtplanbarkeit und nur eingeschränkte Steuerbarkeit, zunehmende Vielfalt und Heterogenität der beteiligten Akteure, nichtlineare Verlaufsmuster sowie hochgradige Kontext- und Interaktionsabhängigkeit. Wesentliche Elemente dieses Innovationsverständnisses lassen sich auch in der „Europäischen Vision der Forschungsund Innovationspolitik im XXI. Jahrhundert" (Europäische Kommission 1998) wiederfinden. Eckpunkte eines zeitgemäßen Innovationsverständnisses sind:

- Interdisziplinarität, Heterogenität, Rekursivität und Reflexivität der Innovationsprozesse;
- Betonung geschichtlicher, kultureller und organisatorischer Voraussetzungen; - Verstärkte Einbeziehung der Nutzer/ Anwender/Bürger in Prozesse einer auf „social pull“ und „public-policy-drive“ gegründeten „Ko-Entwicklung“;

- Fokussierung auf Innovationssysteme im Sinne von miteinander verbundenen Institutionen und Akteuren, die in einem interaktiven Prozess Wissen, Fähigkeiten und Artefakte schaffen, speichern und transferieren;

- Notwendigkeit der Koordination verschiedener am Innovationsgeschehen beteiligter Akteursgruppen;

- „Hybridisierung“ sowohl an der Grenze Gesellschaft (Praktiker/Nutzer) - Wissenschaft (Experte/Entwickler) als auch an der Grenze (weiche) Sozialwissenschaft - (harte) Ingenieurs-/Naturwissenschaft.

Ziele, Prozesse, Strukturen, Akteure sowie Bewertungsmaßstäbe unterscheiden sich hier deutlich vom industriegesellschaftlich geprägten Paradigma: Gesellschaftliche Ziele wie Beschäftigung und Lebensqualität stehen im Zentrum. Lineare Modelle werden zugunsten von systemischen, interaktiven Modellen abgelöst. An die Stelle eines stark auf die Rolle der Wissenschaft als Innovationstreiber fokussierten Modells, auf dem bis heute zahlreiche große politische Förderprogramme basieren, tritt ein Modell, in dem die Gesellschaft selbst zum Ort von Innovationen wird. Mit diesem veränderten Verständnis der Innovationsprozesse treten neue Akteure in den Mittelpunkt des Interesses. Bullinger spricht in diesem Zusammenhang von einem „Paradigmenwechsel des Innovationssystems" (Bullinger 2006, S. 14). „An die Stelle der traditionellen großen Unternehmen und staatlichen Forschungseinrichtungen treten flexible Innovationsnetzwerke" (ebd.). Dabei kommt den Nutzern eine zunehmend wichtige Rolle zu. „Ein großer Teil industrieller Innovation wird nicht von den Herstellern geschaffen, sondern durch

\footnotetext{
2 Der von Davids Mitte der 1990er Jahre eingeführte Begriff der Pfadabhängigkeit bezeichnet den Umstand, dass die Entwicklungsvergangenheit einer Organisation, eines Produktes, einer Technologie etc. künftige Entwicklungsmöglichkeiten beeinflusst (Blättel-Mink 2006, S. 98).

3 Zu Reichweite und Grenzen kontextualistischer Ansätze der Innovationsforschung vgl. Fichter 2003.
} 
die Nutzer der Produkte initiiert" (ebd.). Burmeister/Neef bringen es auf den Punkt, wenn sie sagen: „Innovationen entziehen sich immer deutlicher dem Korsett einer technisch verkürzten Sichtweise und einem eng verstandenen unternehmerischen Kontext. Innovationen benötigen Gesellschaft und Gesellschaft benötigt Innovationen" $(2003$, S. 3$)$.

\section{Bedeutungswandel und -zuwachs von sozialen Innovationen}

Dennoch wird Innovation auch in den Sozialwissenschaften nach wie vor asymmetrisch gedacht. Der Fokus richtet sich primär auf technologische Innovationen (Rammert 1997, S. 3). Soziale Innovationen werden hingegen vorwiegend in ihrem funktionalen Verhältnis zu diesen bzw. als deren „kompensatorisches Gegenstück“ verstanden (Braun-Thürmannn 2005, S. 25); als eigenständiges Phänomen werden sie kaum systematisch behandelt.

\subsection{WAS SIND SOZIALE INNOVATIONEN?}

Nach einer Definition von Gillwald sind soziale Innovationen gesellschaftlich folgenreiche, vom vorher gewohnten Schema abweichende Regelungen von Tätigkeiten und Vorgehensweisen. Sie sind überall in gesellschaftlichen Systemen möglich, im Ergebnis Verhaltensänderungen und verwandt, aber nicht gleich, mit technischen Innovationen" (Gillwald 2000, S. 1). Zapf definiert soziale Innovationen als „neue Wege, Ziele zu erreichen (...), die die Richtung des sozialen Wandels verändern". Sie „können Voraussetzungen, Begleitumstände oder Folgen technischer Innovationen sein. “ Ihre Produktivität hängt „,von der menschlichen Kreativität und von symbolischen Ressourcen ab." Ebenso wie technische Innovationen beruhen sie auf „wissenschaftlichem Fortschritt wie auf praktischer Erfahrung" (Zapf 1989, S. 177f.).

Es handelt sich mithin um einen spezifischen, von technischen Innovationen abgrenzbaren und unterscheidbaren Innovationstypus. Die Tatsache der prinzipiellen sozialen Einbettung einer jeden Innovation führt dementsprechend keines- wegs „dazu, dass jede Innovation, selbst wenn sie im Kern technologischer Natur ist, eine soziale Innovation ist" (Vordank 2005, S. 43).

Vor dem Hintergrund dieser Entwicklungen gibt es vielfältige Gründe, sich mit dem Thema "soziale Innovationen“ intensiver zu beschäftigen. Zapf hob in seinem Aufsatz in der Sozialen Welt aus dem Jahre 1989 die Bedeutung der Innovationstheorie und -forschung sowohl für die Gesellschaftstheorie als auch für die Sozialwissenschaften als Profession hervor, weil sie „Entwicklung als einen inhärent konfliktreichen, ungleichgewichtigen, unsicheren Suchprozess begreifen" (ebd. S. 178) und „auf dieser Grundlage sozialen Wandel mit der Kombination von technischen und sozialen Innovationen als Prozess der schöpferischen Zerstörung erklärbar machen" (ebd. S. 181).

\subsection{STARKE IMPULSE AUS DEM DIENSTLEISTUNGSBEREICH}

Wir gehen davon aus, dass starke Impulse zur Durchsetzung eines neuen Innovationsverständnisses von der wachsenden wirtschaftlichen und beschäftigungspolitischen Bedeutung des Dienstleistungssektors, in dessen Mittelpunkt soziale Innovationen stehen, ausgehen werden. Bereits heute sind annähernd drei von vier Erwerbstätigen in der Dienstleistungswirtschaft beschäftigt. Mit zunehmender Bedeutung des Dienstleistungssektors gegenüber dem produzierenden Sektor könnten sich die Vorzeichen von Innovationsprozessen umkehren. Innovationen sind dann nicht mehr allein neue Produkte und neue Technologien, ${ }^{4}$ sondern vor allem eine Neukonfiguration sozialer Arrangements. Soziale Innovationen könnten dann zum vorrangigen Zielpunkt von Innovationsprozessen werden. Innovation kann insofern „als ein Prozess kollektiver Schöpfung verstanden werden, in dessen Verlauf die Mitglieder einer bestimmten Gesamtheit neue Spielweisen für das soziale Spiel der Zusammenarbeit und des Konflikts, mit einem Wort eine neue Praxis, erlernen, d. h. erfinden und festlegen, und in dessen Verlauf sie sich die dafür notwendigen kognitiven, relationalen und organisatorischen Fähigkeiten aneignen" (Crozier/Friedberg 1993, S. 19).

Die Bedeutung sozialer Innovationen für den wirtschaftlichen relevanten Verwertungsprozess wird weiter zunehmen.
Wenn im Bereich des produzierenden Gewerbes die technischen Innovationen als neue Produkte zentrale Zielpunkte des Innovationsprozesses darstellen und Prozessinnovationen Mittel zur effizienteren Herstellung von entsprechenden Produkten sind, so stehen im Dienstleistungsbereich soziale Innovationen als neue "Produkte“ im Fokus. Die zunehmende wirtschaftliche Bedeutung des Dienstleistungsbereiches dürfte in den nächsten Jahren dazu beitragen, dass die vielleicht entscheidende Ursache für das Schattendasein sozialer Innovationen, im Vergleich zu den naturwissenschaftlich-technischen Entwicklungen, erodieren wird. Braun-Thürmanns Feststellung, mit sozialen Innovationen ,ist kaum ökonomischer Gewinn zu erzielen, was dazu führt, dass sie in einer Gesellschaft, die sich oftmals in den Kategorien von wirtschaftlichem Erfolg bzw. Misserfolg beschreibt, eher im Randbezirk des öffentlich-politischen Interesses stehen " (Braun-Thürmanns 2005, S. 25), könnte angesichts ihrer wachsenden Bedeutung beim Ausbau der wirtschaftlichen Leistungsfähigkeit von Unternehmen und Regionen bald der Vergangenheit angehören.

\subsection{DIENSTLEISTUNGSINNOVATIO- NEN UND SOZIALE INNOVATIONEN}

Weil Erzeugung und Verbrauch insbesondere von personen-, aber auch von unternehmensbezogenen Dienstleistungen meist zeitlich zusammenfallen (Uno-actu-Prinzip), und dementsprechend der Integrationsgrad der Konsumenten, ihrer (veränderten) Lebensbedingungen, Verhaltensweisen, Normen und Werte höher als bei der Erstellung materieller Güter ist, sind soziale und Dienstleistungsinnovationen inhaltlich und unter dem Gesichtspunkt ihrer Genese eng miteinander verwoben. „Dabei spielt auch die Verknüpfung von Produkten und Dienstleistungen zu einem integrierten Gesamtkonzept eine entscheidende Rolle. Den Kundinnen und Kunden werden auf diese Weise innovative Dienstleistungspakete in Kombination mit komplexen Technologien und Produkten angeboten“" (Kutzner 2007, S. 28).

4 „Innovationen - das sind neue Produkte, Technologien und technische Verfahren, mit denen wir Märkte erschließen und zukunftssichere Arbeitsplätze schaffen“ (Edelgard Bulmahn). 
Wie eng soziale und Dienstleistungsinnovationen konkret miteinander verwoben sind, zeigt sich z. B. deutlich bei der Herausbildung von neuen nutzungsdauerverlängernden (Reparatur, Wiederverwendung) und nutzungsintensivierenden (Miete, gemeinschaftliche Nutzung) Nutzungsregimen und damit einhergehenden veränderten Verhaltensweisen einerseits und neuen (ökologisch vorteilhaften) Dienstleistungsangeboten andererseits (Hirschel et al. 2001; Konrad/Nill 2001). Für komplexe Systeminnovationen (z. B. im wachstumsintensiven Bereich energieeffizienter Technologien, Architektur, Sanierung, Ingenieurleistung und darauf bezogener Forschung, Entwicklung, Vernetzung und Beratung) ist die Interdependenz von technischen, organisatorischen und sozialen Innovationen kennzeichnend. Systeminnovationen erfordern weitreichende evolutionäre Transformationsprozesse und soziale Innovationen unter intelligenter Nutzung neuer Technologien und Dienstleistungen. Sie beruhen auf der „Zusammenarbeit unterschiedlicher Leistungsanbieter. Dies setzt voraus, dass Unternehmen gemeinsame Visionen entwickeln, Allianzen schließen, Vereinbarungen treffen und komplexe Beziehungsnetzwerke managen “ (Bierter 2001, S. 12). Dies gilt in besonderer Weise für „Nachhaltigkeitsinnovationen" bzw. für die damit zusammenhängenden Anforderungen an Steuerungsund Koordinationsformen, wie sie z. B. unter Stichworten wie Transition-Management, Transdisziplinarität, „Beyond Technological Innovation“ oder „Modelling of Technological Change“ (ISI/DIW 2004) diskutiert werden.

\subsection{TECHNISCHE UND SOZIALE INNOVATIONEN}

Die grundlegenden Unterschiede zwischen technischen und sozialen Innovationen sowohl in ihrem Charakter als auch in ihren Verbreitungsformen, Wirkungsmechanismen und Akteurskonstellationen werden bislang kaum thematisiert, geschweige denn erforscht. Genau hier aber liegt ein Schlüssel zum vertieften Verständnis eines neuen Innovationsparadigmas, welches soziale Innovationen als die eigentlichen Motoren der Innovationsdynamik moderner Gesellschaften begreift.

In der Debatte um soziale Innovation ist die Frage ihrer Bewertung von besonderer Bedeutung. Sind soziale Innovationen per definitionem ,gut“, „sozial erwünscht“ etc. oder können sie in ihren Wirkungen ebenso wie technische Innovationen - negative, unerwünschte Auswirkungen haben? In der Diskussion zu den Folgen technischer Innovationen übernehmen soziale Innovationen in der Regel die Funktion, negative Auswirkungen abzufedern bzw. sozialverträglich zu gestalten. In diesem Kontext werden sie meist mit normativen Konnotationen verwendet. Jenseits derartiger Zuschreibungen gilt bei sozialen wie bei technischen Innovationen, dass wir uns von einem naiven Innovationsverständnis verabschieden sollten, das Innovationen per se als positiv und wünschenswert definiert und die Risiken und Folgeprobleme ausblendet, um sie der sozialverträglichen Nachbearbeitung zu überlassen.

Das postindustrielle Innovationsparadigma muss diese Widersprüche in angemessener Weise thematisieren, um sie im Sinne einer "reflexiven Modernisierung" bearbeitbar zu machen (Beck 1986). Die Analyse dieser Ambivalenzen und die Entwicklung differenzierter Gestaltungskonzepte stellt eine Zukunftsaufgabe sozialwissenschaftlicher Forschung dar.

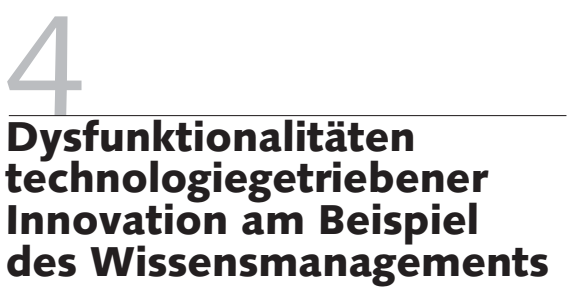

\subsection{EXZESSIVES WISSENS- MANAGEMENT - FRUSTRIERTE HOFFNUNGEN}

Wie wir im Rahmen eines vom Bundesministerium für Bildung und Forschung (BMBF) geförderten Projekts im Bereich der wissensintensiven Dienstleistungen am Beispiel des Themas „Wissensmanagement" aufzeigen konnten, führt ein verengtes technologieorientiertes Innovationsverständnis zu Fehlentwicklungen und Dysfunktionalitäten. ${ }^{5}$ So wurde in einer ersten Wissensmanagementgeneration versucht, Probleme der Wissensorganisation in Unternehmen und Netzwerken durch ambitionierte Technikentwicklungen zu bewältigen. Diese technikgetriebenen Lösungen enttäuschten jedoch in der Praxis. Wissensmanagement wandelte sich in der betrieblichen Wahrnehmung vom Hoffnungsträger zur Frustrations- erfahrung (Howaldt et al. 2004; Heisig 2005).

Der technologische Impetus vieler Wissensmanagementprojekte der ersten Generation führte $\mathrm{zu}$ einem Wissensmanagementverständnis, welches vor dem Hintergrund der neuen technologischen Möglichkeiten die umfassende Sammlung, Aufbereitung und Verwaltung des Wissens in den Mittelpunkt der Aufmerksamkeit rückte. In vielen Unternehmen und Netzwerken stand das Ziel im Mittelpunkt, personengebundenes implizites Wissen in digitaler Form aufzubereiten, zu speichern und für die Organisation als Ganzes verfügbar zu halten. Diese Prozesse der Sammlung, Aufbereitung und Speicherung von Wissen gingen nicht nur mit hohen zeitlichen und finanziellen Aufwänden einher. Aufgrund der damit verbundenen Dekontextualisierung verwandelte sich ein Großteil dieses Wissens in mehr oder weniger nutzlose Daten und Informationen.

Zudem wurden wertvolle Ressourcen auf den Umgang mit bereits vorhandenem, zum Teil bereits veraltetem Wissen konzentriert, anstatt auf die Kreation von neuem Wissen. Diese Entwicklungen haben die Ausgangsprobleme in den Unternehmen, die zur Einführung von Wissensmanagementlösungen führten (Intransparenz über die Informations- und Wissensbasis, hoher Suchaufwand, Probleme des Nachvollzugs der Inhalte, keine Wirksamkeit bei der Mobilisierung impliziten Wissens), häufig verschärft und zu einer chaotisch-exzessiven Daten- und Wissensarchivierung (mit dem dazugehörigen Pflegeaufwand) geführt.

Unsere sozialwissenschaftliche Analyse konnte zeigen, dass vor dem Hintergrund

\footnotetext{
5 Das Forschungs- und Entwicklungsvorhaben "cross company knowledge management" (crosscomp) ist im Kontext des Rahmenkonzepts "Innovative Arbeitsgestaltung - Zukunft der Arbeit" vom BMBF in den Jahren 2001 bis 2004 gefördert worden. Im Projekt wurde der Frage nach den Besonderheiten des Wissensmanagements in Netz werken wissensintensiver Dienstleistungsunternehmen (Beratungsbranche, IT- und Medien) nachgegangen. Neben umfangreichen Internetund Literaturrecherchen umfasste das Untersuchungskonzept Experteninterviews und 19 Fallstudien in Deutschland, Österreich, der Schweiz und den USA sowie die Konzipierung und Durchführung zweier Modellprojekte in Beratungs- und IT-Netzwerken (Howaldt et al. 2004).
} 
der scheinbar unbegrenzten Möglichkeiten neuer technologischer Entwicklungen zur Speicherung und Aufbereitung von Wissen die Besonderheiten der Ressource Wissen (Personen- und Kontextgebundenheit, Dynamik) grob vernachlässigt wurden (Stehr 2001, S. 62). Die Ressource Wissen wurde technologiekompatibel zugerichtet und, in eine organisierte, geordnete verwalt- und archivierbare Form gezwungen" (Baecker 2000, S. 107), während „soziale Aspekte“ des Wissensmanagements (Kommunikationsstandards, Regelsysteme, Motivation, Kompetenzen) und nichttechnisierbare Formen des Wissensaustauschs (Umgang mit personalem und organisationalem implizitem Wissen) weitgehend ausgeblendet blieben.

\subsection{WISSENSMANAGEMENT ALS SOZIALE INNOVATION}

Die Aufarbeitung organisations-, wissensund techniksoziologischer Befunde ermöglichte eine Kritik dieser verkürzten, als „exzessiv" zu charakterisierenden Form des Wissensmanagements, welche eine Hauptursache für das Scheitern vieler Implementationsversuche offenlegte.

Gleichzeitig konnte mit dem „selektiven Wissensmanagement" ein Alternativkonzept konturiert werden, das die Besonderheiten der Ressource Wissen berücksichtigt und Wissensmanagement als die Gesamtheit der personalen, organisatorischen, kulturellen und technischen Praktiken definiert, die in einer Organisation bzw. in einem Netzwerk auf eine effiziente Nutzung der Ressource „Wissen“ zielen. Die Grundannahmen des selektiven Wissensmanagements sind denen des exzessiven Wissensmanagements diametral entgegengesetzt. So richtet das selektive Wissensmanagement den Fokus auf Zusammenhänge zwischen Wissen und sozialem Handeln sowie auf die Transformation von Wissen in Nutzen. Die zentrale Aufgabe des Wissensmanagements liegt in der Entwicklung von Selektionsmechanismen, die den Handelnden eine Konzentration auf das für die Organisation relevante Wissen ermöglichen. Methodisch und konzeptionell setzt der Ansatz auf den Ausbau von Kommunikationsbeziehungen, die den direkten Wissensfluss zwischen den Wissensträgern ermöglichen und so Chancen für gemeinsame Lern- und Kommunikationsprozesse bieten. $\mathrm{Zu}$ solchen Kommunikationsprozessen gehören ebenso
face-to-face-Formen wie auch virtuelle Gemeinschaften via Internet bzw. Intranet. IT-Technologien dienen vorwiegend als Kommunikationsmedien, die eine Vernetzung der Wissensträger erlauben. Auf leistungsfähige Archive und Datenbanken sowie technische Programme wird damit zwar nicht verzichtet, aber sie werden radikal auf ihre Unterstützungsfunktion verwiesen. Entscheidende Wissensträger bleiben Personen.

In den Erhebungen von Crosscomp zeigte sich eine große Übereinstimmung im Hinblick auf die Bedeutung unmittelbarer Kommunikation von Wissensträgern in konkreter Projektarbeit, auf Treffen von Netzwerkpartnern, in themenzentrierten temporären Arbeitsgruppen. Die Ursache der Wertschätzung direkter Kommunikation liegt in ihrem Beitrag zur Aktivierung des für Arbeits- und Innovationsprozesse unverzichtbaren impliziten Wissens. Anstatt dem bisherigen Dogma zu folgen und implizites Wissen aufwendig in explizites Wissen zu verwandeln, um es für technologieunterstützte Dokumentationsprozesse verwertbar zu machen, folgt selektives Wissensmanagement der Idee, die Technologieunterstützung zur Identifikation von Know-how-Trägern mit ihren Erfahrungsund Wissensprofilen zu nutzen. In dieser Perspektive geht es dann darum, diese Experten auch über die eigenen Organisationsgrenzen hinweg ausfindig zu machen und so zu vernetzen, dass ihre (impliziten) Wissensbestände um ein konkretes Problem herum mobilisiert und integriert werden.

Im Kern lassen sich an diesem Beispiel sowohl die Dysfunktionalitäten eines Innovationsmodus technologisch getriebener Sozialanpassung wie die Leistungsfähigkeit eines auf soziale Innovation fokussierten Innovationsmodus aufzeigen. Die Möglichkeiten der neuen Technologien werden hierbei nicht ausgeblendet. Ihr Einsatz wird aber durch die konkreten Zielstellungen und Konzeptionen eines neuen sozialen Kommunikationszusammenhangs bestimmt.

In der Analyse der Ursachen des Scheiterns setzte sich die Einsicht durch, dass die Einführung von Wissensmanagement als soziales Phänomen bearbeitet werden muss. Inzwischen bildet sich eine zweite, durchaus Erfolg versprechende Wissensmanagementphase heraus, in der technologische Angebote in die kontextuellen Vorgaben und individuellen Bedürfnis- lagen der sozialen Akteure einbettet sind (Pawlowski et al. 2007; BMWi 2007).

Die neue Generation des Wissensmanagements kommt nicht mehr als überzogene technologische Heilsverkündung daher, sondern als aushandlungs- und entscheidungsbasierter Organisationsprozess, dessen Kommunikationserfordernisse durch einen Instrumentenmix bewältigt werden, in dem Technologieunterstützung optional und relational gehalten wird. Will Technologieentwicklung nicht ins Leere laufen, muss sie ihre Anschlussfähigkeit an konkrete soziale Praxen sicherstellen. ${ }^{6}$

\section{Zur neuen Rolle der Sozialwissenschaften}

Welche Rückschlüsse lassen sich aus den bisherigen Ausführungen für die Rolle der Sozialwissenschaften im Innovationsgeschehen ziehen? Mit Blick auf die Bedeutung sozialer Innovationen kommt Zapf zu dem Schluss, dass die sozialwissenschaftlich generierten „Werkzeuge der Entscheidungshilfe (...) sehr wohl die Problemlösungs- und Steuerungsfähigkeit moderner Gesellschaften erhöhen“, dass die Sozialwissenschaften wichtige Funktionen bei der aktiven Gestaltung konkreter Innovationsprozesse übernehmen und damit eine erhöhte Relevanz im Innovationsgeschehen beanspruchen können (Zapf 1989, S. 183f.). Mit der Herausbildung der Wissensgesellschaft nehmen die Anforderungen an die Gestaltungskompetenz der Sozialwissenschaften zu.

Die sozialwissenschaftliche Forschung hat in den letzten Jahren viel dazu beigetragen, die sozialen Voraussetzungen von Innovationen und den sozialen Charakter von Innovationsprozessen herauszuarbeiten. In dieser Analyse liegt sicherlich ihre Stärke. Die hier gewonnenen Erkenntnisse sind inzwischen tief in das gesellschaftliche Bewusstsein eingedrungen und bestimmen

\footnotetext{
6 Im Hinblick auf den Zusammenhang zwischen so zialer Innovation und neuen Dienstleistungsangeboten sei darauf verwiesen, dass angelehnt an diesen Innovationstyp neue Beratungsangebote für Unternehmen entwickelt wurden, die insbesondere auf kleine und mittelständische Unternehmen zielen.
} 
das Denken und Handeln der relevanten gesellschaftlichen Akteure. ${ }^{7}$

Trotz ihrer Bedeutung bei der Konturierung eines neuen Innovationsmodells hat es die Sozialwissenschaft jedoch nicht geschafft, als gleichbedeutender Innovationsgestalter neben den Natur- und Ingenieurwissenschaften Anerkennung zu finden und dementsprechend in einschlägigen Förderprogrammen berücksichtigt zu werden. Ein wenig ratlos versuchen die Sozialwissenschaften, wenigstens den Fuß in die Tür der Innovationsforschung und -politik zu bekommen, indem sie sich „als Begleitforschung (...) in Form einer unterstützenden Funktion einer technologiebasierten Infrastruktur- und Wirtschaftsförderpolitik ins Gespräch (...) bringen“ (Aderhold 2005, S. 16).

Diese Position bleibt auf merkwürdige Weise hinter eigenen Forschungsergebnissen zurück und spiegelt damit ähnlich defensive Routinen unterschiedlichster Akteure in der Innovationsdebatte wider: Von der Wahrnehmung und analytischen Durchdringung aktueller Innovationsbedingungen bis zur konsequenten Ausrichtung des eigenen Handelns scheint es noch ein langer Weg zu sein. Die Beobachtung veränderter Bedingungen der Wissensorganisation (Nowotny et al. 2001; Weingart 2001) und die Aufwertung des Sozialen im Innovationsprozess korrespondieren noch längst nicht mit der Einsicht, dass das neue Innovationsmodell auch die Operationslogik der Sozialwissenschaften unter Innovationsdruck setzt (Bullinger/Bienzeisler 2007). Dies übersieht jedoch die Gefahr eines weiteren Bedeutungsverlustes im Innovationsgeschehen - und damit auch im Prozess der gesellschaftlichen Modernisierung.

Die Sozialwissenschaften haben den Innovationsprozess neu interpretiert. Offen bleibt jedoch die Frage nach ihrem Beitrag zur Entwicklung und Gestaltung von Innovationen. Allein auf die Analysefähigkeit begrenzte Ansätze sozialwissenschaftlicher Forschung erweisen sich hinsichtlich ihres eigenen Rollenverständnisses, ihrer Routinen und Methodiken der Wissensgenerie- rung als extrem zurückhaltend gegenüber Veränderungen. Der vornehme Rückzug in methodologischen Konventionalismus und kritisch-analytische Begleitung technologisch getriebener „Innovationsangebote" als einzig wahre Aufgabe der Sozialwissenschaften verschließt sich den Chancen einer Neupositionierung als aktiver Innovationstreiber in einem Innovationsgeschehen, an dem die potenziellen Anwender/Kunden nicht als Endstufe eines linearen Entwicklungsprozesses erscheinen, sondern in hochgradig interaktive Kommunikationsnetzwerke als aktive Problemlöser und Innovatoren eingewoben werden. Angesichts der komplexen Wissensverarbeitungsprozesse in derartigen Netzwerken greifen Bemühungen zur Verfeinerung der konventionellen Transferlogik zu kurz. Die Erforschung, Entwicklung und Gestaltung dieser Prozesse steht noch an ihrem Anfang (Ciesinger et al. 2005). Die Sozialwissenschaften sind für diese Aufgabe prädestiniert, können ihr aber nur gerecht werden, wenn sie sich selbst aktiv als „enabler" bzw. Dienstleister neuer Formen der Wissensproduktion in einschlägigen Netzwerken bewegen.

Die Kernkompetenz der Sozialwissenschaften im Innovationsgeschehen liegt in der Gestaltung sozialer Kontexte, in denen der Wissensaustausch zwischen Problemlösern, Experten, Key-Usern, Anwendern verschiedener gesellschaftlicher Teilsysteme stattfinden kann und Lernprozesse initiiert werden können (Gustavsen 2006).

Der besondere Charakter sozialwissenschaftlichen Wissens, welcher lange Zeit vor dem Hintergrund scheinbarer naturwissenschaftlicher Exaktheit und Verlässlichkeit als Mangel empfunden wurde ${ }^{8}$ wird vor dem Hintergrund des neuen Innovationsparadigmas zu einer zentralen Stärke. Sozialwissenschaftliches Reflexionswissen kann einen wichtigen Beitrag zur Erhöhung der Fähigkeit zur Selbstbeobachtung und Selbstreflexion der Gesellschaft leisten (Howaldt 2004, S. 44ff.). Diese Fähigkeit zur Selbstbeobachtung und Selbstreflexion ist jedoch, ebenso wie die Einbindung zentraler gesellschaftlicher Ak- teure, eine Voraussetzung, um die immanente Ambivalenz und das Risiko von Innovationsprozessen durch den Einbau von Reflexionsschleifen abzufedern. Sie machen die Frage nach der Umsetzung von „Erfindungen“, neuen Ideen in konkrete Innovationen zum Gegenstand von Entscheidungen und durchbrechen damit den „Fortschrittsautomatismus" (Was neu ist, ist gut) des alten Paradigmas. In Abgrenzung zu one-best-way- bzw. one-singletruth-Ansätzen kann Wissensproduktion, die auf die Einbeziehung aller relevanten Akteure von der Idee bis zur Umsetzung setzt, einem neuen Innovationsparadigma konkrete Gestalt geben. Die Auflösung konventioneller Strukturen der Arbeitsteilung zwischen Wissensproduzenten und Wissensanwendern, zwischen Entwicklern und Anwendern, die immer stärkere Einbindung des Erfahrungswissens der Peripherie in der Wissensgenerierung, verweisen auf zunehmende Interaktivitätsanforderungen von Innovationsprozessen. Soziale Innovationen bedürfen in besonderem Maße gelingender Kommunikation, Kooperation und Wissensintegration zwischen heterogenen Akteuren. „Vor diesem Hintergrund ist die Bedeutung sozialwissenschaftlicher Expertise für die Analyse und Gestaltung von Innovationsprozessen nicht obsolet, sondern aktueller denn je“ (Bullinger/Bienzeisler 2007, S. 57). Für die Disziplin ergeben sich Anlass wie Chance, ihre Rolle im Modernisierungsprozess neu zu definieren.

\footnotetext{
7 Dabei scheinen sich im Kontext der Innovationsforschung die Thesen von Beck und Bonß aus den 1980 er Jahren zum Prozess der Diffusion sozialwissenschaftlicher Erkenntnisse in die Praxis zu bestätigen. Die Sozialwissenschaften erweisen sich hier als wichtiger Lieferant von Erkenntnissen, die in das gesellschaftliche Bewusstsein eindringen und die darauf bezogenen Konzepte der Politik und Wirtschaft zur Steuerung und zum Management solcher Innovationsprozesse nachhaltig prägen, ohne jedoch als Disziplin davon zu profitieren (Beck/Bonß 1989).

8 Vgl. kritisch hierzu: Daston/Galison 2007.
} 
Aderhold, J. (2005): Gesellschaftsentwicklung am Tropf technischer Neuerungen, in: Aderhold, J./John, R. (Hrsg.): Innovation. Sozialwissenschaftliche Perspektiven, Konstanz, S. 13-32

Baecker, D. (2000): Die "andere Seite" des Wissensmanagements, in: Götz, K. (Hrsg.): Wissensmanagement: Zwischen Wissen und Nichtwissen, München, S. 99-112

Beck, U. (1986): Risikogesellschaft. Auf dem Weg in eine andere Moderne, Frankfurt am Main

Beck, U./Bonß, W. (Hrsg.) (1989): Weder Sozialtechnologie noch Aufklärung? Analysen zur Verwendung sozialwissenschaftlichen Wissens, Frankfurt a. M.

Bierter, W.(2001): Zukunftsfähiges System-Design, Institut für Produktdauer-Forschung/Factor 10 Innovation Network, Genf-Giebenbach (Manuskript)

Blättel-Mink, B. (2006):Kompendium der Innovationsforschung, Wiesbaden

Braun-Thürmann, H. (2005): Innovation, Bielefeld

Bullinger, H.-J. (2006): Verdammt zur Innovation, in: RKW-Magazin 57, S. $12-14$

Bullinger, H.-J./Bienzeisler, B. (2007): Innovation und hybride Wertschöpfung, in: profile, Internationale Zeitschrift für Veränderung, Lernen, Dialog 13, S. 54-58

Bundesministerium für Wirtschaft und Technologie (BMWi) (2007):

Wissensmanagement in kleinen und mittleren Unternehmen und öffentlichen Verwaltungen. Ein Leitfaden, Berlin

Burmeister, K./Neef, A. (2003): Innovate - don't imitate, in: changeX Partnerforum [28.07.2003], www.changex.de

Chesbrough, H. W. (2003): Open Innovation. The New Imperative for Creating and Profiting from Technology, Boston

Ciesinger, K.-G./Howaldt, J./Klatt, R./Kopp, R. (2005) (Hrsg.): Modernes Wissensmanagement in Netzwerken - Perspektiven, Trends und Szenarien, Wiesbaden

Crozier, M./Friedberg, E. (1993): Die Zwänge kollektiven Handelns Über Macht und Organisation, Frankfurt am Main

Daston, L./Galison, P. (2007): Objektivität, Frankfurt a. M.

Europäische Kommission (1998): Die Gesellschaft, letzte Grenze. Eine europäische Vision der Forschungs- und Innovationspolitik im XXI. Jahrhundert, Brüssel-Luxemburg

Fichter, K. (2003): Kontextsteuerung. Potenziale eines gesellschaftstheoretischen Steuerungskonzepts für das Innovationsmanagement, Borderstep-Arbeitspapier 1, Berlin

Fichter, K./Noack, T./Beucker, S./Bierter, W./Springer, S. (2006): Nachhaltigkeitskonzepte für Innovationsprozesse, Stuttgart

Gillwald, K. (2000): Konzepte sozialer Innovation, in: WZB paper: Querschnittsgruppe Arbeit und Ökologie, Berlin

Gustavsen, B. (2006): Innovation and action research, in: International Journal of Action Research 3, S.267-289
Heidenreich, M. (1997): Zwischen Innovation und Institutionalisierung. Die soziale Strukturierung technischen Wissens, in: Blättel-Mink, B./Renn, O. (Hrsg.): Zwischen Akteur und System. Die Organisation von Innovation, Opladen, S. 177-206

Heisig, P. (2005): Stand und Zukunft des Wissensmanagements, in: Ciesinger, K.-G./Howaldt, J./Klatt, R./Kopp, R. (Hrsg.): Modernes Wissensmanagement in Netzwerken - Perspektiven, Trends und Szenarien, Wiesbaden, S. 71-90

Hirschel, B./Konrad, W./Scholl, G. U./Zundel, S. (2001): Nachhaltige Produktnutzung. Sozial-ökonomische Bedingungen und ökologische Vorteile alternativer Konsumformen, Berlin

Howaldt, J. (2004): Neue Formen sozialwissenschaftlicher Wissensproduktion in der Wissensgesellschaft. Forschung und Beratung in betrieblichen und regionalen Innovationsprozessen, Münster

Howaldt, J./Klatt, R./Kopp, R. (2004): Neuorientierung des Wissensmanagements, Wiesbaden

Fraunhofer-Institut für System- und Innovationsforschung (ISI)/Deutsches Institut für Wirtschaftsforschung (DIW) (2004): Proceedings zum Workshop Nachhaltigkeit und Innovation. Diskussionsstand und Wirtschaftswissenschaftlicher Forschungsbedarf, 12. -13. 2., im Rahmen des Sondierungsprojektes "Nachhaltige Entwicklung und Wirtschaftswissenschaften, Berlin

Konrad, W./Nill, J. (2001): Innovationen für Nachhaltigkeit. Ein interdisziplinärer Beitrag zur konzeptionellen Klärung aus wirtschafts- und sozialwissenschaftlicher Perspektive, Schriftenreihe des IÖW 157/01, Berlin Kutzner, E. (2007): Glück auf - der Service kommt, in: Sozialforschungsstelle Dortmund: Jahresbericht 2006, Dortmund, S. 28-34

Nowotny, H./Scott-Morgan, P./Gibbons, M. (2001): Re-Thinking Science. Knowledge and the Public in an Age of Uncertainty, Cambridge Pawlowski, P./Gerlach, L./Hauptmann, S./Puggel, A. (2007): Wissen als Wettbewerbsvorteil in kleinen und mittelständischen Unternehmen, Chemnitz

Rammert, W. (1997): Innovation im Netz. Neue Zeiten für technische Innovationen: heterogen verteilt und interaktiv vernetzt, in: Soziale Welt 48 , S. $397-416$

Reichwald, R./Piller, F. T. (2005): Open Innovation. Kunden als Partner im Innovationsprozess, München

Stehr, N. (2001): Wissen und Wissenschaften, Frankfurt a. M.

Vordank, T. (2005): Zur organisationalen Reziprozität von Diffusion, in: Aderhold, J./John, R. (Hrsg.): Innovation. Sozialwissenschaftliche Perspektiven, Konstanz, S. 33-48

Weingart, P. (2001): Die Stunde der Wahrheit. Zum Verhältnis der Wissenschaft zu Politik, Wirtschaft und Medien in der Wissensgesellschaft, Weilerswist

Zapf, W. (1989): Über soziale Innovation, in: Soziale Welt 1/2, S. 170183 